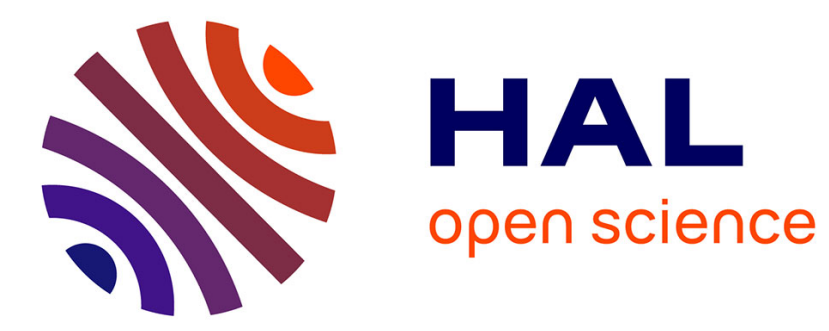

\title{
Considerations on the Relevance of Cerebral Fusiform Aneurysms Observed During HIV Infection
}

Bruno Law-Ye, Robert-Yves Carlier, Raphaël Richard, Raphaël Blanc, Claire Jourdan, Pierre de Truchis, Flore Viry, Didier Dormont, Delphine Leclercq, Frédéric Clarençon

\section{To cite this version:}

Bruno Law-Ye, Robert-Yves Carlier, Raphaël Richard, Raphaël Blanc, Claire Jourdan, et al.. Considerations on the Relevance of Cerebral Fusiform Aneurysms Observed During HIV Infection. Clinical Neuroradiology, 2017, pp.1-9. 10.1007/s00062-017-0581-0 . hal-01520254

\section{HAL Id: hal-01520254 https://hal.sorbonne-universite.fr/hal-01520254}

Submitted on 10 May 2017

HAL is a multi-disciplinary open access archive for the deposit and dissemination of scientific research documents, whether they are published or not. The documents may come from teaching and research institutions in France or abroad, or from public or private research centers.
L'archive ouverte pluridisciplinaire HAL, est destinée au dépôt et à la diffusion de documents scientifiques de niveau recherche, publiés ou non, émanant des établissements d'enseignement et de recherche français ou étrangers, des laboratoires publics ou privés. 


\section{Considerations on the Relevance of Cerebral Fusiform Aneurysms observed during HIV infection}

Bruno Law-ye ${ }^{1,2}$, Robert-Yves Carlier ${ }^{3,4,5}$, Raphaël Richard ${ }^{3}$, Raphaël Blanc ${ }^{6}$, Claire Jourdan $^{7}$, Pierre de Truchis ${ }^{8}$, Flore Viry ${ }^{9}$, Didier Dormont ${ }^{1,2}$, Delphine Leclercq ${ }^{1}$ and Frédéric Clarençon ${ }^{1,2}$

${ }^{1}$ APHP, Neuroradiology Department, Hôpital Pitié-Salpêtrière, Paris, France

${ }^{2}$ Sorbonne Universités, Pierre et Marie Curie Faculty of Medicine, Paris, France ${ }^{3}$ APHP, Radiology Department, Hôpital Raymond Poincaré, Garches, France

${ }^{4}$ Paris Ile-de-France Ouest Faculty of Medicine, Versailles, Saint-Quentin-en-Yvelines ${ }^{5}$ CIC 1429, Raymond Hôpital Raymond Poincaré, Garches, France

${ }^{6}$ APHP, Neuroradiology Department, F. Ophtalmologique Adolphe de Rotschild, Paris, France

${ }^{7}$ APHP, Rehabilitation Department, Hôpital Raymond Poincaré, Garches, France ${ }^{8}$ APHP, Infectious Diseases Department, Hôpital Raymond Poincaré, Garches, France ${ }^{9}$ APHP, Radiology Department, Hôpital Saint-Antoine, Paris, France 


\begin{abstract}
HIV-associated ectatic cerebral vasculitis (HIV-AECV) is a rare vasculitis with diffuse fusiform aneurysms. Its pathophysiology remains poorly understood. Though extensively described in children, it is still incompletely studied in adults. Our objective was to present 5 adult cases with emphasis on imaging findings and long-term evolution. From 2006 to 2014, we included 5 HIV-infected patients presenting fusiform cerebral aneurysms. Vessels abnormalities were assessed with brain CT-angiography, MR-angiography and/or digital subtraction angiography.

All patients had brain MR assessment. Clinical and biological data were analysed. Fusiform aneurysms of carotid terminations extending to middle and anterior cerebral arteries were bilateral in 3 patients and unilateral in one. More distal fusiform aneurysms were observed in four patients and saccular aneurysms in two patients. Two patients suffered from ischemic lesions while none experienced haemorrhage.

Unlike recent reviews, our study underlines the low haemorrhagic potential of HIV-AECV; long-term follow-up suggest a monophasic evolution under antiretroviral medication.
\end{abstract}




\section{Key words:}

Human immunodeficiency virus, acquired immunodeficiency syndrome, cerebral vasculitis, fusiform aneurysm, circle of Willis, CT, MRI, DSA

\section{Abbreviations and acronyms:}

$\mathrm{CT}=$ Computerized tomography

$\mathrm{CTA}=\mathrm{CT}$ angiography

DSA $=$ Digital subtraction angiography

$\mathrm{HCV}=$ Hepatitis $\mathrm{C}$ virus

$\mathrm{HIV}=$ Human immunodeficiency virus

HIV-AECV $=$ HIV-related associated cerebral vasculitis

ICA = Internal carotid artery

MRA = Magnetic resonance angiography

MRI = Magnetic resonance imaging 


\section{Introduction}

Ectatic vasculitis is a rare form of human immunodeficiency virus (HIV)-induced angiitis. Though more than 30 cases HIV-associated ectatic cerebral vasculitis (HIV-AECV) have been reported in children since the 1980's [1-4], only few data are available on HIV-AECV in adults (mainly case reports [1] or short cases series $[5,6]$ ). The most commonly described pattern of HIV-AECV in children is fusiform arterial ectasia involving the internal carotid artery (ICA) terminus and the ipsilateral M1 and A1 segments [7].

We aimed to evaluate through 5 consecutive patients from 4 University Hospitals, the clinical symptoms, immunological and viral data, imaging findings and clinical/radiological long-term outcome in adult patients with HIV-AECV and review the literature. 


\section{Materials and Methods}

From October 2006 to April 2014, we retrospectively included HIV-infected patients with cerebral ectatic vasculitis from 4 different tertiary centres for HIV-related brain disease.

Inclusion criteria were as follows:

- Documented HIV infection

- Fusiform aneurysms of the circle of Willis documented either by Computed Tomography Angiography (CTA), Magnetic Resonance Angiography (MRA) or Digital Subtraction Angiography (DSA) or a combination of these exams Exclusion criteria consisted of another cause of vasculitis (from inflammatory disease or from another infectious causes), HIV patients with only saccular intracranial aneurysms and/or patients aged less than 18 years.

We included five consecutive patients ( 5 females; mean age at time of diagnosis $=31.4 \pm 9.5$; range 23-47 years).

\section{Clinical assessment}

Clinical symptoms and revealing conditions like headache, seizure, intracranial bleeding (parenchymal/pericerebral), acute ischemic stroke were systematically recorded by a senior neurologist.

\section{$H I V$-related data assessment}

Viral and immune data from the patients were assessed from their medical chart. The following data were systematically recorded: mode of HIV transmission (maternal-foetal transmission, acquired contamination (sexual, transfusion, toxic abuser), CD4 count $\left(\right.$ cells $\left./ \mathrm{mm}^{3}\right)$ and viral replication $\left(\right.$ copies $\left./ \mathrm{mm}^{3}\right)$ at the time of diagnosis and HIV-related opportunistic infections. 


\section{Imaging explorations}

Four patients $(80 \%)$ underwent CTA; 4 patients $(80 \%)$ a 3D time of flight (TOF) MRA and 2 (40\%) DSA. Three patients (60\%) had a combination of these modalities (Patients \#1, \#2 and \#5). In most cases, brain MR was performed with 3 Tesla MRI scanner (General Electric, Medical systems, Milwaukee, Wisc). The imaging protocol included T2 FLAIR (fluidattenuated inversion-recovery)-weighted, T1-weighted, diffusion-weighted, T2-weighted gradient echo (GE), 3D-time of flight angiography and contrast enhanced T1 SE sequences.

CTAs were mainly performed with a 16-slice CT scanner (Siemens Medical Solutions, Erlangen, Germany).

On angiographic evaluation, location of the vasculitis on the circle of Willis (i.e. the segment involved according to the Fischer's segmentation [8] and its extent (number of segment involved, unilateral/bilateral involvement) were systematically assessed. Presence of additional saccular aneurysms was also recorded on vascular imaging. All the images were reviewed in consensus by 2 senior neuroradiologists with an experience in neuroradiology of 7 and 2 years, respectively, on the PACS (Picture Archiving Communicating System) workstation (Agfa, Mortsel, Belgium).

Associated findings brain abnormalities on CT and MRI were systematically recorded (i.e.: subarachnoid haemorrhage, intraparenchymal hematoma, acute ischemic stroke, mass effect, brain or brain stem compression). Thrombosis of the aneurysms, calcifications and wall enhancement were also systematically recorded on MRI and/or CTA.

\section{Clinical/imaging follow-up:}

Clinical follow-up was performed by the physician in charge (AIDS specialist, rehabilitator and/or neurologist): occurrence of a new neurological deficit, clinical symptoms suggestive of 
intracranial haemorrhage and signs of compressive syndrome (on brainstem and/or cranial nerves) were systematically assessed. On imaging follow-up, aneurysm's shape and size changes, haemorrhagic or ischemic lesions and opportunistic infections were systematically recorded. Monitoring time of vascular abnormalities was variable depending on the centre and the patients' status and symptoms. Mean length of clinical and imaging follow-ups was recorded.

Management of HIV-AECV was systematically recorded (i.e: conservative, surgical and/or endovascular treatment).

\section{Ethical statement}

Neither approval of the institutional review board nor patient informed consent are required by the ethics committee of the institutions involved in this series for retrospective analysis of patients' records and imaging data. 


\section{Results}

\section{Clinical symptoms/revealing conditions}

Clinical presentations were status epilepticus (Patient \# 1), headache (\# 2), generalized seizures (\# 3), and meningo-encephalitis (\# 5). It was fortuitously diagnosed in Patient \# 4 who suffered from toxoplasmosis (Table 1). No patient presented intracranial bleeding as a revealing symptom.

Various neurological deficits were recorded: pharmaco-resistant epilepsy and neuropsychological deficits (Patient \# 1), retinal necrosis with severe visual deficits (\# 2), transient left hand hemiparesis due to a right lenticular infarct (\# 3) and psychomotor retardation (\# 5). Patient \# 4 had no permanent neurological deficits.

\section{$H I V$-related data}

\section{HIV infection}

HIV contamination was maternal-foetal in 2 patients and acquired at adult age in 3 patients presumably from sexual transmission. Four patients were of subsaharan African origin and one of Maghreb origin. Diagnosis was made from routine pregnancy assessment in 2 patients. Mean length of evolution of the infection from the time of the diagnosis was $14.2 \pm 9.2$ years (range 5-25 years).

All patients experienced poor treatment adherence with consecutive intense viral replication and low TCD4 (Table 1). 


\section{Opportunistic infections}

Poor viral control and immunosuppression were evidenced from opportunistic infections that affected all patients, such as pneumocystosis, brain toxoplasmosis, CMV retinitis, zoster, uterus cervix dysplasia and peri-anal condylomas and candidosis (Table 1). Patient \# 1 also had an HCV co-infection.

\section{Imaging findings}

1. Vessels abnormalities (see Table 2)

All patients had an involvement of anterior circulation with fusiform aneurysm of the internal carotid artery (ICA) termination extending to proximal A1 and/or M1 segments of the anterior and the middle cerebral arteries (ACA, MCA), respectively. It was unilateral in two patients (Fig. 1) and bilateral in 3 patients (Fig. 2). Supra-cavernous ICA was impaired in all cases while cavernous ICA was involved in only two patients. Additional fusiform aneurysms were observed in Patients \# 1, 3, 4 and 5 (Fig. 1). Saccular aneurysms were observed in Patients \# 1 (Fig. 3) and \# 5. In Patient \# 1, a partially thrombosed giant vertebral (V4 segment) aneurysm was observed (Fig. 4).

Involvement of posterior circulation was observed only in Patient \# 1, consisting of irregular fusiform dilatations of intracranial vertebral arteries, basilar and posterior cerebral arteries (in addition to the saccular LVA aneurysm). In the remaining cases, vertebrobasilar system was notably spared.

Aneurysm's wall calcifications were observed in three patients. Arterial wall enhancement was seen in three patients, suggesting vessel wall inflammation (Fig. 5). Finally, no evidence of aneurysm rupture was recorded. 
2. Ischemic/hemorrhagic lesions

Two patients suffered from ischemic lesions (Patients \# 1 and 2) confirmed on DWI: right lenticulo-caudate, left thalamic and right temporal symptomatic infarcts in \# 1 and right lenticular infarct in \# 2. No patient presented haemorrhagic lesions.

\section{Clinical/imaging follow-up}

During follow-up, no patient presented signs of subarachnoid haemorrhage. In all cases, fusiform aneurysms remained stable on angiographic follow-up. Patient \# 1 had a 17-yearlong follow-up without any modification of the fusiform aneurysms. However, in this patient, a saccular left vertebral aneurysms progressively increased in size over 6 years, compressing the brainstem and required exclusion treatment. Patient \# 5 also had steady aneurysmal disease over a 17-year period (Table 2).

\section{Treatment}

All patients had instauration/optimization of antiretroviral therapy at diagnosis of brain vasculopathy. They did not have further treatment like steroids or cytotoxic drugs.

No exclusion treatment was performed regarding fusiform aneurysms. Patient \# 1 had an endovascular treatment of a symptomatic left V4 giant aneurysm (Fig. 4) by parent artery occlusion with coils. Follow-up MRI showed a regression of both the aneurysm and its mass effect, leading to clinical improvement. 


\section{Discussion}

We here-in report 5 new cases of HIV-AECV, among the largest original case series in adults [5,9]. A first adult case was reported in 2006 [1] and only 32 adult cases had been reported since then [5,9]. A recent cohort study reported 20 patients with similar findings among a cohort of more than $6000 \mathrm{HIV}$-infected patients [9]. However, this study did not to focus specifically on follow-up and on hemorrhagic risk associated with these fusiform aneurysms. HIV-AECV usually affects immunocompromised patients with T CD4 lymphocytes counts $<200 / \mathrm{mL}$ and a viral load $>100000$ copies $/ \mathrm{mm}^{3}$. In the literature, mean time between infection and central nervous system events is 6.8 years in children $[0.17-12.6]$ and 10.3 years in adults [7-12]. The most frequent modes of HIV transmission were perinatal or following blood transfusion in children and perinatal or unknown in adults [5]. These data are consistent with the ones of our patients, who had a long-term HIV infection (mean evolution length: $12.4 \pm 9.2$ years [range: $5-25$ years]); two patients presenting a maternal-foetal HIV contamination. All patients suffered from immunosuppression due to poor treatment adherence and follow-up.

According to a recent review of the literature, seven cases of brain infarction, five cases of subarachnoid haemorrhage and one case of intracerebral hemorrhage in patients with HIVAECV have been reported so far [5].

In our series, we observed two cases $(40 \%)$ of brain infarcts but did not record any haemorrhagic event, despite a long-term follow-up.

Conversely, the above-mentioned recent review focused on brain aneurysms in HIV patients reported a higher risk of haemorrhage: $9.1 \%$ in children and $43.8 \%$ in adults [5].

This discrepancy with our results may be explained by the fact that nearly all the patients who suffered from haemorrhage collected in this review [5] bled from an associated saccular intracranial aneurysm and not from the fusiform aneurysm(s). 


\section{Pathophysiology}

The pathophysiology of HIV-AECV is complex and not yet entirely elucidated. Histopathology shows a thickening of the vessel wall with media and adventice inflammation fibrous and collagenous media thickening and endothelium mesenchymal proliferation [10]. Pathomechanisms potentially include direct infection of the vessel wall and perivascular tissues [11] as well as an indirect role of the HIV through complex immune reactions involving CD8 lymphocytes, superantigens and dysregulation of adhesion molecules and cytokins. Some authors reported evidences of vessel wall infection [4] while others did not [3].

Extracranial and intracranial HIV-associated vasculopathy may result from different mechanisms [12]. Low T CD4 lymphocytes are associated with intracranial vasculopathy in which the intima could be the main target, with inflammation triggered by transendothelial migration of the virus. The disease may then progress by vascular remodelling, growth factors and cytokines, likely induced by opportunistic infections [12].

Immune reconstitution inflammatory syndrome (IRIS) was suggested as a possible cause [2], but remains controversial and does not seem incriminated in our patients, since the vasculopathy occurred early after the diagnosis of the infection. A high dose of azidothymidine and varicella-zoster virus vasculitis have been suspected in paediatric cases $[13,14]$

\section{Imaging findings}

We observed a specific pattern of fusiform aneurysms involving the ICA terminations and extending contiguously to the proximal segments of the anterior and middle cerebral arteries. This pattern is similar to the one previously described in paediatric cases $[3,4,7,15,16]$. It is 
noteworthy that some authors have reported HIV-vasculitis with more distally located intracranial aneurysms [17].

\section{Differential diagnoses}

Differential diagnoses mainly include spontaneous fusiform aneurysms. Their main causes are dissection and atherosclerosis and they frequently provoke ischemic symptoms. They may involve vertebrobasilar circulation and compress the brainstem as well [18]. Unlike saccular aneurysms, fusiform aneurysms rarely rupture due to their stiff, thickened wall [19].

Rare cases of diffuse fusiform cerebral aneurysms associated with dysimmune conditions have been reported and seem to bring further arguments regarding the role of immunodeficiency and inflammation: diffuse aneurysms secondary to chronic Epstein Barr virus meningitis in a patient with immune deficit [20], vertebrobasilar and ICA aneurysms associated with varicella angiitis in a patient with immunodeficiency and T-cell dysfunction [3] and finally, progressive bilateral fusiform ICA aneurysms in a child with T-cell immunodeficiency [21].

Unless having a histological demonstration of HIV infection of the vessel wall, HIV direct role in the formation of these fusiform aneurysms remains putative. HIV-infected patients without neurological symptoms are usually not systematically screened by brain CT or MR angiography. Such a screening strategy could be useful to diagnose these abnormalities earlier and to better understand their pathogenesis, especially to demonstrate the correlation between viral load and low CD4 T lymphocytes and the appearance or worsening of the vasculopathy. However, some previous reports have incriminated HIV as a certain pathogenic agent for fusiform aneurysms, especially with histological evidences of HIV within vessel walls and secondary wall inflammation [4]. Moreover, the existence of numerous paediatric cases reported in the literature is likely to rule out an atherosclerotic origin of these aneurysms. 
Though even rarer, similar vascular patterns have been depicted in patients infected with other pathogenic agents such as EBV [20], underlying the fact that fusiform aneurysms are not pathognomonic of HIV vasculitis. Additionally, co-infection (i.e.: HIV + other opportunistic infection[s]), either synchronous or successive, might be responsible for the formation of these intracranial vascular changes. Indeed a case of HIV-RECV has been reported in a child who presented a co-infection by HIV and VZV [13].

Finally, some anti-viral medications are also known to cause atherosclerotic changes that might participate to the genesis of these fusiform aneurysms. However, in most of our patients, the aneurysms were discovered prior to anti-viral therapy introduction or after a long period of treatment interruption.

\section{Treatment}

Although HIV-AECV has important clinical implications, the optimal treatment strategy is debated [17-19]. HIV-related vasculitis is usually depicted as a monophasic affection when treated with antiretroviral therapy. Objectives are both to treat the vasculitis and control the infection. Thus, the treatment is mainly based on plasma exchange and antiretrovirals, with avoidance of cytotoxic agents and corticosteroids. However some authors recommend the use of corticosteroids, following the treatment of any active infection and the initiation of antiretroviral therapy $[18,19]$.

Our patients had optimized antiretroviral treatment. None of them had steroids or cytotoxic medication or plasma exchange.

Recently, endovascular treatment of such fusiform aneurysms in HIV-AECV has been reported, suggesting that flow diversion may be a suitable treatment option. However, such procedures carry significant complication risk and remain controversial [22,23]. Indeed, the literature carries no strong evidence of a bleeding risk from the fusiform aneurysms in HIV- 
AECV patients. As well, in our series, no haemorrhagic event was recorded over a 9.25 years $[1-17]$ mean follow-up delay.

\section{Study limitations}

One limitation of our study is the heterogeneity of the clinical and radiological follow-up. This heterogeneity can be explained by the fact that the patients were treated in different institutions, with different follow-up protocols and by the fact that some patients came from foreign countries. Additionally, very long-term follow-up was not available in all patients. However, we do believe that reporting our experience of a very low haemorrhagic risk in this disease is pertinent since recent reports of invasive endovascular procedures with significant risks of neurological complications have been published for the treatment of these HIVAECVs [23].

Second, the retrospective design of the study that was responsible for non-homogeneous clinical and angiographic follow-ups and for missing data. 


\section{Conclusion}

Our case series of HIV-AECV in adult patients show that the angiographic pattern consisting of fusiform ectasia involving the ICA terminus extended to both adjacent A1 and M1 segments is very suggestive of the diagnosis. Our study underlines the low haemorrhagic potential of the fusiform aneurysms observed in these HIV patients. Additionally, data from the long-term follow-up suggest a monophasic evolution of this disease under anti-retroviral therapy. These results should be confirmed by larger series, but suggest that conservative management of these HIV-associated fusiform intracranial aneurysms may be a suitable option.

Acknowledgements: The authors would like to thank Dr David Zucman (Internal Medicine, Hôpital Foch, Suresnes, France) for his assistance in the preparation of this manuscript Conflicts of Interest: All of the authors declare they have no conflicts of interest to disclose. 


\section{References}

1. Ake JA, Erickson JC, Lowry KJ. Cerebral aneurysmal arteriopathy associated with HIV infection in an adult. Clin. Infect. Dis. Off. Publ. Infect. Dis. Soc. Am. 2006;43:e46-50.

2. Bonkowsky J, Pavia A. Cerebral vasculopathy with aneurysm formation in HIV-infected young adults. Neurology. 2007;68:623; author reply 623.

3. Daugherty WP, Clarke MJ, Cloft HJ, Lanzino GL. Going viral: fusiform vertebrobasilar and internal carotid aneurysms with varicella angiitis and common variable immunodeficiency. J. Neurosurg. Pediatr. 2009;4:528-31.

4. Dubrovsky T, Curless R, Scott G, Chaneles M, Post MJ, Altman N, et al. Cerebral aneurysmal arteriopathy in childhood AIDS. Neurology. 1998;51:560-5.

5. Baeesa SS, Bakhaidar M, Almekhlafi MA, Madani TA. Human Immunodeficiency VirusAssociated Cerebral Aneurysmal Vasculopathy: A Systematic Review. World Neurosurg. 2016;87:220-9.

6. Modi G, Ranchod K, Modi M, Mochan A. Human immunodeficiency virus associated intracranial aneurysms: report of three adult patients with an overview of the literature. J. Neurol. Neurosurg. Psychiatry. 2008;79:44-6.

7. Carvalho Neto Ad null, Bruck I, Coelho LO, Cruz CR, Liu CB, Gomes AF, et al. [Cerebral arterial aneurysm in a child with acquired immunodeficiency syndrome: case report]. Arq. Neuropsiquiatr. 2001;59:444-8.

8. Fischer E. Die Lageabweichungen der vorderen hirnarterie im gefassbild. Zentralbl Neurochir. 1938;300-13.

9. Edwards NJ, Grill MF, Choi HA, Ko NU. Frequency and Risk Factors for Cerebral Arterial Disease in a HIV/AIDS Neuroimaging Cohort. Cerebrovasc. Dis. Basel Switz. 2016;41:1706.

10. Shah SS, Zimmerman RA, Rorke LB, Vezina LG. Cerebrovascular complications of HIV in children. AJNR Am. J. Neuroradiol. 1996;17:1913-7.

11. Tipping B, de Villiers L, Wainwright H, Candy S, Bryer A. Stroke in patients with human immunodeficiency virus infection. J. Neurol. Neurosurg. Psychiatry. 2007;78:1320-4.

12. Tipping B, de Villiers L, Candy S, Wainwright H. Stroke caused by human immunodeficiency virus-associated intracranial large-vessel aneurysmal vasculopathy. Arch. Neurol. 2006;63:1640-2.

13. Fulmer BB, Dillard SC, Musulman EM, Palmer CA, Oakes J. Two cases of cerebral aneurysms in HIV+ children. Pediatr. Neurosurg. 1998;28:31-4.

14. Lang C, Jacobi G, Kreuz W, Hacker H, Herrmann G, Keul HG, et al. Rapid development of giant aneurysm at the base of the brain in an 8-year-old boy with perinatal HIV infection.

Acta Histochem. Suppl. 1992;42:83-90. 
15. Goldstein DA, Timpone J, Cupps TR. HIV-associated intracranial aneurysmal vasculopathy in adults. J. Rheumatol. 2010;37:226-33.

16. Sedat J, Alvarez H, Rodesch G, Lasjaunias P. Multifocal cerebral fusiform aneurysms in children with immune deficiencies report of four cases. Interv. Neuroradiol. J. Peritherapeutic Neuroradiol. Surg. Proced. Relat. Neurosci. 1999;5:151-6.

17. Hamilton DK, Kassell NF, Jensen ME, Dumont AS. Subarachnoid hemorrhage and diffuse vasculopathy in an adult infected with HIV. Case report. J. Neurosurg. 2007;106:47880 .

18. Echiverri HC, Rubino FA, Gupta SR, Gujrati M. Fusiform aneurysm of the vertebrobasilar arterial system. Stroke. 1989;20:1741-7.

19. Makita K, Tsuchiya K, Furui S, Yoshida H, Yamada H, Takiguchi H. Nondissecting vertebral fusiform aneurysm: embolization using wire-directed detachable balloons. AJNR Am. J. Neuroradiol. 1993;14:340-2.

20. Patil AKB, Azad ZR, Mathew V, Alexander M. Chronic meningitis and central nervous system vasculopathy related to Epstein Barr virus. Ann. Indian Acad. Neurol. 2012;15:303-6.

21. Piantino JA, Goldenberg FD, Pytel P, Wagner-Weiner L, Ansari SA. Progressive intracranial fusiform aneurysms and T-cell immunodeficiency. Pediatr. Neurol. 2013;48:1304.

22. Delgado Almandoz JE, Crandall BM, Fease JL, Scholz JM, Anderson RE, Kadkhodayan $Y$, et al. Successful endovascular treatment of three fusiform cerebral aneurysms with the Pipeline Embolization Device in a patient with dilating HIV vasculopathy. J.

Neurointerventional Surg. 2014;6:e12.

23. Law-Ye B, Dormont D, Chiras J, Carlier R-Y, Clarençon F. Successful endovascular treatment of three fusiform cerebral aneurysms with the Pipeline Embolization Device in a patient with dilating HIV vasculopathy. J. Neurointerventional Surg. 2015; 


\section{Figure captions}

\section{Figure 1.}

A: 3D time of flight (TOF) MRA performed in Patient \# 3; maximum intensity projection (MIP) showing fusiform aneurysm of the left internal carotid artery (ICA) terminus extending to the middle cerebral artery (MCA) and both anterior cerebral arteries (ACAs) (note the hypoplasia of the right A1 segment), faint fusiform ectasia of both A2 segments (arrows) and ectasia of M2 segment. B. Coronal view in MIP reconstruction. Note the fusiform dilatation of the left M2 segment (arrow) and the absence vertebrobasilar system involvement.

\section{Figure 2.}

Digital subtraction angiography (DSA) in right (A) and left (B) oblique anterior projections and $3 \mathrm{D}$ reconstructions $(\mathrm{C}$ and $\mathrm{D})$ performed in Patient \# 4 showing a marked ectasia of the right ICA terminus extending to A1 and M1 segments. Similar pattern, less pronounced, is observed on the left side associated with an aneurysm of the MCA bifurcation.

\section{Figure 3.}

Patient \# 1. A: MRA in coronal MIP reconstruction (performed in 1998): bilateral ectasia of both ICA terminus extending to ipsilateral ACA and MCA is seen. Bilateral ectasia of A2 segments of the ACA is also depicted. B: Follow-up MRA performed in 2012, showing stability of the fusiform aneurysms without any progression. Note the presence of a left saccular carotid-ophthalmic aneurysm.

\section{Figure 4.}

A: MRI sagittal T1-weighted sequence performed in Patient \# 1 at age 33 showing a saccular aneurysm of the left vertebral artery (arrow). B: MRI performed 6 years later showing significant increasing in size of the aneurysm measuring $25 \mathrm{~mm}$ with a mass effect on the bulbo-medullary junction, responsible for headache and gait disorders.

\section{Figure 5.}

A: MRA in Patient \# 2 showing marked ectasia of the right internal carotid extending to A1 and M1 segments (arrow). Blood flow signal is decreased within the fusiform aneurysm's lumen, indicating turbulences related to hemodynamic slow-down. Post-contrast axial (B) and coronal (C and D) T1 SE showing arterial wall enhancement. 